\title{
ATOMIC MEASURE SPACES AND ESSENTIALLY NORMAL COMPOSITION OPERATORS
}

\author{
R.K. Singh and T. Veluchamy
}

The adjoint of a composition operator $C_{T}$ on the $L^{2}$-space of an atomic measure is computed and a characterization for an operator to be a composition operator is given in this short note. The dimensions of kernel and co-kernel of $C_{T}$ are calculated in order to characterise Fredholm composition operators. Finally, essentially normal composition operators are studied on $\imath^{2}$.

\section{Introduction}

Let $(X, S, \lambda)$ be a sigma-finite measure space and $T$ be a nonsingular measurable transformation from $X$ into itself. Then the mapping $C_{T}$ on $L^{p}(\lambda)$ which takes $f$ into $f \circ T$ is a linear transformation. If the range of $C_{T}$ is in $L^{p}(\lambda)$ and $C_{T}$ is bounded, then we call it a composition operator on $L^{p}(\lambda)$ induced by $T$. It is known that the composition transformation $C_{T}$ is bounded if and only if there exists an $M>0$ such that $\lambda T^{-1}(E) \leq M \lambda(E)$ for all $E$ in $S$. From this it follows that if $C_{T}$ is bounded then the induced measure $\lambda T^{-1}$ is absolutely continuous with respect to the measure $\lambda$. Hence, by the Radon-Nikodym theorem, there exists a positive measurable function $f_{0}$

Received 18 November 1982. 
such that $\lambda T^{-1}(E)=\int_{E} f_{0} d \lambda$ for every $E$ in $S$. The function $f_{0}$ is called the Radon-Nikodym derivative of the measure $\lambda T^{-1}$ with respect to $\lambda$.

A measurable set $E$ is called an atom if $\lambda(E) \neq 0$ and if $F \in S$ and $F \subset E$, then either $\lambda(F)=0$ or $\lambda(E)=\lambda(E)$. A measure $\lambda$ is called atomic if every element $E \in S$ such that $\lambda(E) \neq 0$ contains an atom and in this case we say that $(X, S, \lambda)$ is an atomic measure

space. In this paper the adjoint of a composition operator on $L^{2}(\lambda)$ is obtained and the necessary and sufficient condition for an operator on $L^{2}(\lambda)$ to be a composition operator is discussed when the underlying measure $\lambda$ is atomic. Also dimensions of kernel of $C_{T}$ and kernel of $C_{T}^{*}$ are given. Finally essentially normal composition operators on $z^{2}$ are characterised.

\section{Composition operators and atomic measure spaces}

If $(X, S, \lambda)$ is a sigma-finite atomic measure space, then we can write $X$ as $\bigcup_{i=1}^{\infty} E_{i}$, where the $E_{i}^{\prime}$ 's are disjoint atoms of finite measure [7]. These atoms are unique in the sense that if $X=\bigcup_{i=1}^{\infty} F_{i}$, where the $F_{i}$ 's are disjoint atoms of finite measures, then for every $F_{i}$ there exists an $E_{j}$ such that $\lambda\left(E_{j} \Delta E_{i}\right)=\lambda\left\{\left(E_{j} \backslash F_{i}\right) \cup\left(E_{i} \backslash E_{j}\right)\right\}=0$. If a non-singular measurable transformation $T$ on $X$ takes one part of an atom $E_{j}$ to a subset of an atom $E_{k}$ and the other part of $E_{j}$ to a subset of another atom $E_{1}$, then anyone of the above parts of $E_{j}$ has to be a null set. As it is obvious that the image of an atorn under a nonsingular transformation cannot be a null set, we can consider a nonsingular measurable transformation $T: X \rightarrow X$ as a transformation taking atoms into atoms. Hereafter we denote the atom $E_{j}$ by $j$ and by $T(j)$ the atom to which $E_{j}$ is carried over by $T$. We say that an atom $j$ is 
in the range of $T$ if $j \in\{T(i): i \in N\}$.

A non-singular measurable transformation $T: X \rightarrow X$ is called one-toone almost everywhere if the inverse image of every atom under $T$ contains at most an atom. It is called onto almost everywhere if the inverse image of every atom under $T$ contains at least one atom. If $T$ is one-to-one almost everywhere and onto almost everywhere then it is called invertible almost everywhere. Also every function $f \in L^{2}(\lambda)$ is constant almost everywhere on an atom. Hence the span of the characteristic functions $\left\{x_{i}: i \in N\right\}$ form a dense subset of $L^{2}(\lambda)$. Let $K_{i}=X_{i} / \lambda(i)$. Then the set of functions $\left\{K_{i}: i \in \mathbb{N}\right\}$ forms an orthonormal basis for $L^{2}(\lambda)$. The symbol $B(H)$ stands for the $C^{*}$-algebra of all bounded operators on the Hilbert space $H$. Throughout this paper we assume that $(X, S, \lambda)$ is an atomic sigma-finite measure space. The following theorem computes the adjoint of $C_{T}$.

THEOREM 1. Let $C_{T} \in B\left(L^{2}(\lambda)\right)$ and let $A$ be defined as $(A f)(i)=1 / \lambda(i) \int_{T^{-1}(i)} f d \lambda$ almost everywhere for $f \in L^{2}(\lambda)$ and for every atom $i$. Then $A=C_{T}^{*}$.

Proof. Let $f, g \in L^{2}(\lambda)$. Then

$$
\begin{aligned}
\left\langle C_{\vec{T}} f, g\right\rangle & =\int_{x}\left(C_{T} f\right) \bar{g} d \lambda \\
& =\sum_{i=1}^{\infty} \int_{T^{-1}(i)} f \circ T \bar{g} d \lambda \\
& =\sum_{i=1}^{\infty} f(i) \lambda(i)(\overline{A g})(i) \\
& =\langle f, A g\rangle .
\end{aligned}
$$

Hence $A=C_{T}^{*}$. Hence the proof is completed.

The following theorem gives a necessary and sufficient condition for an operator to be a composition operator. 
THEOREM 2. Let $A \in B\left(L^{2}(\lambda)\right)$. Then $A$ is a composition operator if and only if the set $\left\{K_{i}: i \in \mathrm{N}\right\}$ is invariant under $A^{*}$. In this case $T$ is determined by $A^{*}\left(K_{i}\right)=K_{T(i)}$.

Proof. The proof follows from the above theorem and [3].

The above theorem shows that the functions $\left\{K_{i}\right\}$ play the role of kernel functions for $L^{2}(\lambda)$. In the following theorem we compute the dimension of $\operatorname{ker} C_{T}$.

THEOREM 3. Let $C_{T} \in B\left(L^{2}(\lambda)\right)$. Then dim ker $C_{T}$ equals the number of atoms in $X \backslash\{T(i): i \in N\}$.

Proof. If an atom $i$ is not in the range of $T$, then $X_{i} \in \operatorname{ker} C_{T}$ since $\lambda T^{-1}(i)=0$. If an atom $j$ is in the range of $T$, then $\lambda T^{-1}(j)>0$ and hence $C_{T^{X}} X_{j} \neq 0$. Hence ker $C_{T}$ is equal to the closure of the span of the set $\left\{X_{k}: k\right.$ is not in the range of $\left.T\right\}$. Hence the proof is completed.

Let $\beta_{n}$ denote one less than the number of atoms in $T^{-1}(n)$ if $T^{-1}(n)$ has more than one atom, otherwise zero.

THEOREM 4. Let $C_{T} \in B\left(L^{2}(\lambda)\right)$. Then dim ker $C_{T}^{*}=\sum_{n=1}^{\infty} \beta_{n}$.

Proof. From Theorem 2 it follows that

$$
C_{T}^{*}\left(\sum_{i \in T^{-1}(k)} a_{i} X_{i}\right)=\left(\sum_{i \in T^{-1}(k)} b_{i}\right) x_{k} \text {, where } b_{i}=\frac{\lambda(i)}{\lambda(T(i))} a_{i} .
$$

From this it is clear that when the cardinality of $T^{-1}(k)=p>1, C_{T}^{*}$ kills $(p-1)$ basis vectors of the closed subspace spanned by the characteristic functions $\left\{X_{i}: i \in T^{-1}(k)\right\}$. Since $L^{2}(\lambda)$ is the direct sum of such closed subspaces, we get dim ker $C_{T}^{*}=\sum_{n=1}^{\infty} \beta_{n}$. Hence the 
theorem is proved.

DEFINITION. An operator $A \in B(H)$ is called Fredholm if $A$ has closed range and dimensions of kernel of $A$ and co-kernel of $A$ are finite.

Let $X_{0}=\left\{x: x \in X\right.$ and $\left.f_{0}(x)=0\right\}$. The following theorem gives a characterization for Fredholm composition operators.

THEOREM 5. Let $C_{T} \in B\left(L^{2}(\lambda)\right)$. Then $C_{T}$ is Fredholm if and only if $f_{0}$ is bounded coway from zero on the complement of $X_{0}$, range of $T$ contains all but finitely many atoms of $X$ and $T$ is one-to-one almost everywhere on the complement of a set with finitely many atoms.

Proof. The proof follows from Theorems 3 and 4 .

SOME CONSEQUENCES

1. $C_{T}$ is an injection if and only if $T$ is onto almost everywhere.

2. $C_{T}$ has dense range if and only if $T$ is one-to-one aimost everywhere.

3. $C_{T}$ is invertible if and only if $T$ is invertible almost everywhere and $f_{0}$ is bounded away from zero.

4. If $C_{T} \in B\left(z^{2}\right)$, then $C_{T}$ is Fredholm if and only if $f_{0}=1$ except for a finite number of points of $\mathbf{N}$.

In [6] it has been proved that in case of a general finite measure space unitary and normal composition operators coincide and isometries and quasinormal composition operators coincide on $B\left(L^{2}(\lambda)\right)$. If the measure space is atomic, then all the above coincide.

THEOREM 6. Let $(X, S, \lambda)$ be a finite atomic measure space and $c_{T} \in B\left(L^{2}(\lambda)\right)$. Then the following are equivalent:

(i) $C_{T}$ is unitary;

(ii) $C_{T}$ is normal; 
(iii) $C_{T}$ is an isometry;

(iv) $C_{T}$ is quasinormal;

(v) $C_{T}$ is a co-isometry.

Proof. By Theorems 1 and 2 of [6], (i) and (ii) are equivalent, and (iii) and (iv) are equivalent. Now suppose $C_{T}$ is an isometry. Then $T$ is measure preserving and hence $\lambda T^{-1}(i)=\lambda(i)$ for every atom $i$ in $X$. Let $S_{i}$ denote the set of all atoms in $X$ which have the same measure as $i$. Then each $S_{i}$ will be a finite set. Also $C_{T}$ is an isometry implies that $T$ is onto almost everywhere. Since $T\left(S_{i}\right) \subset S_{i}$ for every $i$, $T / S_{i}$ is one-to-one almost everywhere and hence $T$ is one-to-one almost everywhere. Since an isometry has closed range, this implies that $C_{T}$ is invertible and hence $C_{T}$ is unitary. This gives the equivalence of $(i)$ and $(i i i)$. To prove the equivalence of $(v)$ and $(i)$, suppose $C_{T}$ is a coisometry. Then $C_{T}$ has dense range and hence $T$ is one-to-one almost everywhere. Also $f_{0} \circ T=1$ almost everywhere. This implies $\lambda T^{-1}(T(i))=\lambda(i)=\lambda(T(i))$ for every $i$ in $X$. Considering the set $S_{i}$ as above we have $T\left(S_{i}\right) \subset S_{i}$ for every $i$ in $X$ which implies that $T$ is onto almost everywhere. Hence $C_{T}$ is invertible and hence $C_{T}$ is unitary. This completes the proof of the theorem.

COROLLARY 6.1. If the atoms in the finite atomic measure space $(X, S, \lambda)$ are such that $\lambda(i) \neq \lambda(j)$ when $i$ is different from $j$, then all the above composition operators coincide with the identity operator.

\section{Essentially normal composition operators on $z^{2}$}

DEFINITION. Let $H$ be a Hilbert space, $C(H)$ denote the ideal of compact operators in $B(H)$ and $\pi$ from $B(H)$ to the Calkin algebra $B(H) / C(H)$ be the canonical epimorphism. Then an operator $A$ in $B(H)$ is said to be essentially normal, essentially unitary or an essential isometry if $\pi(A)$ is normal, unitary or an isometry respectively in the $C^{*}$-algebra 
$B(H) / C(H)$ (refer [1]). We say that $A$ is quasi-unitary if $A^{*} A-I$ and $A A^{*}-I$ are finite rank operators. $A$ is Fredholm is equivalent to saying that $\pi(A)$ is invertible in the Calkin algebra.

We know from [4] that invertible, normal, unitary and isometric composition operators are not different in $l^{2}$. The same is true about Fredholm, essentially normal, essentially unitary and essential isometric composition operators. This we shall exhibit in the following theorem.

THEOREM 7. Let $C_{T} \in B\left(z^{2}\right)$. Then the folzowing are equivalent:

(i) $C_{T}$ is Fredholm;

(ii) $C_{T}$ is essentially normal;

(iii) $C_{T}$ is quasi-unitary;

(iv) $C_{T}$ is essentially unitary;

(v) $C_{T}$ is an essential isometry.

Proof. First we prove the equivalence of $(i)$ and $(i i)$. Let $C_{T}$ be Fredholm. Then $f_{0}=1$ except for a finite number of points of $N$. Now

$$
C_{T}^{*} C_{T}-C_{T} C_{T}^{*}= \begin{cases}M_{f_{0}-f_{0} \circ T} & \text { on } \overline{\operatorname{Ran} C_{T}} \\ M_{f_{0}} & \text { on } \operatorname{ker} C_{T}^{*}=\left(\operatorname{Ran} C_{T}\right)^{\perp} .\end{cases}
$$

Since $C_{T}$ is Fredholm $f_{0}=f_{0} \circ T$ except for a finite number of points of $\mathrm{N}$ and $\operatorname{ker} C_{T}^{*}$ is finite dimensional. Hence $C_{T}^{*} C_{T}-C_{T} C_{T}^{*}$ is of finite rank and hence $C_{T}$ is essentially normal. Conversely, suppose $C_{T}$ is essentially normal. If possible let dimension of $\operatorname{ker} C_{T}=\infty$. Then by Theorem 3 there exists an infinite subset $s_{1}=\left\{n_{1}, n_{2}, \ldots\right\}$ of $N$ such that $f_{0}\left(n_{i}\right)=0$ for $i=1,2, \ldots$. For $n \in N$ let $e^{(n)}$ be the function on $N$ taking value zero at points different from $n$ and value 1 at $n$. Then the sequence $\left\{e^{(n)}\right\}$ tends to zero weakly. But the sequence 
$\left\{\left\|\left(C_{T}^{*} C_{T}-C_{T} C_{T}^{*}\right) e^{(n)}\right\|\right\}$ does not tend to zero. This shows that $\left(C_{T}^{*} C_{T}-C_{T} C_{T}^{*}\right)$ is not compact. Thus $C_{T}$ is not essentially normal which is a contradiction. Hence dim ker $C_{T}<\infty$. Similarly, if $\operatorname{ker} C_{T}^{*}$ is infinite dimensional, then we can prove that $\left(C_{T}^{*} C_{T}-C_{T} C_{T}^{*}\right)$ is not compact. Hence dim ker $C_{T}^{*}<\infty$. Thus $C_{T}$ is Fredholm, since every composition operator on $l^{2}$ has closed range.

If $C_{T}$ is Fredholm, then $f_{0}=1$ except for a finite number of points of $N$. Hence $C_{T}^{*} C_{T}-I=M_{f_{0}^{-1}}$ is of finite rank. Also

$$
C_{T} C_{T}^{*}-I= \begin{cases}M_{f_{0} \circ T-1} \text { on } \overline{\operatorname{Ran} C_{T}}, \\ -I \quad & \text { on }\left(\widetilde{\operatorname{Ran} C_{T}}\right)^{\perp}=\operatorname{ker} C_{T}^{*} .\end{cases}
$$

Since $f_{O} \circ T=1$ except for a finite number of points of $N$ and ker $C_{T}^{*}$ is finite dimensional, $C_{T} C_{T}^{*}-I$ is also of finite rank and hence $C_{T}$ is quasi-unitary. Hence (i) implies (iii). Obviously (iii) implies (iv) and (iv) implies (v). Now, let $C_{T}$ be an essential isometry. Then $C_{T}^{\star} C_{T}-I$ is compact which implies $M_{f_{0}-1}$ is compact. This implies $f_{0}=1$ except for a finite number of points of $N$. Hence $C_{T}$ is Fredholm. Thus (v) implies $(i)$ and the theorem is proved.

THEOREM 8. Let $C_{T}$ be a quasinormal composition operator on $l^{2}$. Then $C_{T}$ is essentially normal if and only if it is normal.

Proof. Since a quasinormal composition operator on $z^{2}$ is an injection, it is essentially normal if and only if $\operatorname{ker} C_{T}^{*}$ is finite dimensional. Also $f_{0}=f_{0} \circ T$. This, in the light of Theorem 3 , implies that $C_{T}$ is essentially normal if and only if $\operatorname{ker} C_{T}^{*}$ is zero dimensional. Thus $C_{T}$ is essentially normal if and only if $C_{T}$ is normal. Hence the theorem is proved. 


\section{References}

[1] Ronald G. Douglas, Banach algebra techniques in operator theory (Pure and Applied Mathematics, 49. Academic Press, New York, London, 1972).

[2] Ashok Kumar, "Fredholm composition operators", Proc. Amer. Math. Soc. 79 (1980), 233-236.

[3] Eric A. Nordgren, "Composition operators on Hilbert spaces", Hilbert space operators, 37-63 (Proc. Conf. Long Beach, California, 1977. Lecture Notes in Mathematics, 693. Springer-Verlag, Berlin, Heidelberg, New York, 1978).

[4] R.K. Singh and B.S. Komal, "Composition operators on $Z^{P}$ and its adjoint", Proc. Amer. Math. Soc. 70 (1978), 21-25.

[5] R.K. Singh and Ashok Kumar, "Characterizations of invertible, unitary, and normal composition operators", BulZ. Austral. Math. Soc. 19 (1978), 81-95.

[6] Robert Whitley, "Normal and quasinormal composition operators", Proc. Amer. Math. Soc. 70 (1978), 114-118.

[7] A.C. Zannen, Integration (North Holland, Amsterdam; Interscience, New York, 1967).

Department of Mathematics,

University of Jammu,

Jammu 180001,

India. 